\title{
Effect of Inhalational Anesthetics Exposure on Reproductive Outcomes and its Predictors Among Health Care Personnel in Jimma Zone Public Hospitals, Southwest Ethiopia, 2021: A Comparative Cross-Sectional Study
}

megersa kelbesa olika ( $\nabla$ megersakelbesa@gmail.com )

Jimma University

Zemenu Muluken Dessalegn

Jimma University

Gezahegn Tesfaye Mekonin

Jimma University

Melka Biratu Aboye

Jimma University

Mitiku Birhanu Wedajo

Jimma University

Tajera Tageza llela

Hawassa University

Wondu Reta Demissie

Jimma University

\section{Research Article}

Keywords: Inhalational Anesthetics, exposure, effects, reproductive outcome, Ethiopia

Posted Date: January 24th, 2022

DOI: https://doi.org/10.21203/rs.3.rs-1214023/v1

License: (c) (1) This work is licensed under a Creative Commons Attribution 4.0 International License.

Read Full License 


\section{Abstract \\ Background}

Emerging of anesthetic opens a new era to medical discipline in relieving patient's pain and stress who undergoing surgery but simultaneously exposes the health care personnel working in areas of anesthetic exposure to many adverse health effects including reproductive outcomes effects. Therefore this study aimed to assess the effect of inhalational anesthetics exposure on reproductive outcomes and its predictors among Health care personnel in hospitals of Jimma zone public hospitals.

\section{Methods}

Comparative cross-sectional study was conducted from May 01 to 30, 2021. We approached 483 health care personnel in Jimma zone public hospitals to partake in this study. Of this number, we recruited 292 Health care personnel, comprising 146 exposed health care personnel and 146 unexposed Health care personnel. Structured questionnaires were used to assess the reproductive outcomes. Data were entered into EPI-data version 4.6.1 and exported to SPSS version 24 for analysis. Binary logistic regressions were carried out to identify associated factors with reproductive outcomes. Statistical significance was declared using a $\mathrm{p}$ value $<0.05$.

\section{Results.}

The overall prevalence of bad reproductive outcome status was high (30.8\%) and the prevalence was much higher among exposed HCPs, (20.9\%) when compared to unexposed HCPs (9.9\%)). Among a total of $292 \mathrm{HCPs}$, the likelihood of occurred bad reproductive outcomes was higher among an exposed group $(A O R=3.17,95 \% \mathrm{Cl}: 1.40-7.16)$; smoke cigarettes $(A O R=8.44,95 \% \mathrm{Cl}: 1.93-36.91)$. The occurrence rate of bad reproductive outcome was higher among 30-45h/week exposure (AOR=11.94; 95\%Cl: 1.25-24.95) if separately analyzed among exposed and age of couple above41years among unexposed (AOR=5.87, $95 \% \mathrm{Cl}$ : 1.56-22.06) were significantly associated with bad reproductive outcomes.

\section{Conclusion}

Prevalence of bad reproductive outcomes was higher among exposed HCPs. Hence, it requires attention through creating awareness about the danger of anesthetics exposure in the study setting suggesting the need to further minimize the exposure.

\section{Introduction}

While one of the principal goals of anesthesia is to prevent patients from feeling pain and stress during surgery, the exposure of workers to waste anesthetic gases is a concern, because of the reported adverse 
effects on the health care personnel working in the potential area of inhalation anesthetic exposure (1$3)$. This exposure occurs when inhalational anesthetics gas is released or leaks during medical procedures in the work areas like operating room, recovery room, delivery room, ICU, or other areas where workers may be subject to job-related exposure $(4,5)$.

Globally, it is estimated that more than 200,000 health care personnel may be exposed to waste anesthetics gases annually(6), and mostly Health care personnel like anesthesiologists, nurse anesthetists, specialists (surgical, obstetric, and orthopedic), OR nurses, operating room technicians, postanesthesia care nurses, dentists, veterinarians are potentially exposed to waste anesthetics gases (WAGs) and are at risk of much occupational illness including adverse reproductive effects like spontaneous abortion, preterm delivery, infertility, stillbirth, congenital anomaly, premature rupture of membrane, and delay in conception to health care personnel are the main concern (7-10).

Large epidemiological investigations have indicated that exposure to WAG can increase the risks of chronic diseases (e.g., liver dysfunction and renal insufficiency), on top of spontaneous abortion, and congenital malformation occurring and can decrease the birth rate and increase the stillbirth rate (11)

The study done in Australia shows there was a high prevalence of preterm birth in women exposed to Un scavenged anesthetic gases $7.3 \%$ compared with $5.7 \%$ in the general population(12). Similarly, the study conducted at Sudan Khartoum indicates that there was a high incidence of infertility in operating room personnel $7.35 \%$ compared to $1.47 \%$ in non-exposed health workers. Higher incidence of abortion in operation room nurses (43.3\%) while $14.29 \%$ in non-exposed health workers (13)

The potential victims of this occupational hazard are health care personnel working in operating facilities with no automatic ventilation or scavenging systems or poor condition, recovery rooms/ ICU where gases exhaled by recovering patients not properly vented or scavenged, leakage in the anesthetics breathing circuit but the severity of these anesthetics exposure hazards of adverse health effects vary according to the type of gases used, the length of exposure, and the gas concentrations( $3,14-16)$.

These potential adverse effects due to occupational chronic exposure to these compounds have led public health authorities to publish standards (guideline and protocols) like the use of the latest inhalational agents with minimum effects, adequate ventilation of $\mathrm{OR}$, use of personal protective equipment's (PPEs) and properly disposing of the pollutes/WAGs by scavenging system to the outside of the facilities and also develop technical procedure like sampling methods, active air treatment systems, maintenance procedures and leak test of the machinery used for anesthesia, air suctioning and air conditioning systems and medical surveillance of exposed workers to regulate the possible health effects (17-19).

But these protocols are not implemented in our setup and we expect many associated health problems and high concentration of WAGs or/and their metabolites in the OR air, in the blood and urine of exposed HCPs beyond the recommended level of WHO and still, the status of adverse health effects was undefined in the setting indeed which needs to be explored and adoption of formal practices and 
regulations to reduce ambient air pollution in the working area to safe or minimum levels of exposure. Thus, the present study aimed to assess the impacts of occupational exposure to waste anesthetics gases on health care personnel reproductive health by indirect measures of reproductive outcomes (inability to conceive, spontaneous abortion, delay conception, congenital malformation, stillbirth, sex ratio and compare the effect of inhalational anesthetics exposed HCPs with that of unexposed HCPs

\section{Methods And Materials}

\section{Study Design and Setting}

The facility-based comparative cross-sectional study was conducted at Jimma zone public Hospital from May 1-30 /2021. Jimma zone is located in Oromia Regional State and is situated about $354 \mathrm{kms}$ away from Addis Ababa city, in the Southwest direction. There are eight public hospitals in the Jimma zone. Among eight hospitals, seven of them are governed by Oromia regional state, and each of them has one operation theatre and recovery and no intensive care unit. There is one university hospital that is currently the only referral hospital in the southwestern part of the country. It has 14 major functional areas with potential anesthetics exposure [ 10 operation room theatre, 3 recovery rooms, and 1 main ICU] where health care personnel were potentially exposed to inhalational anesthetics hazards

\section{Population and Sampling}

All health care personnel working in Jimma zone public hospitals involves both exposed and unexposed HCPs with a 1:1 ratio and matched with age group, sex, and professional rank. All HCPs working more than two years and who married/ engaged/coupled at least 1 year ago and who attempted to own children were included and while Health care personnel who has a previous history of spontaneous abortion, fetal defects, low birth weight, detachment, and premature rupture of membrane, pre-term delivery and tested for infertility before entering their present occupation, and all health care personnel these previously worked in OR, ICU, ex-ray room and recovery for more than six months were excluded. A total of 483 HCPs were surveyed. Among only 292 HCPs were reached for final analysis while 191 were excluded from the study based on the exclusion criteria as shown below in (Figure 1).

\section{Data Collection Procedure and Data Quality Control}

A structured questionnaire adapted from the Reproductive Toxicity Risk Assessment tools(20) was used to collect data about the prevalence and factors associated with the effect of anesthetics exposure on reproductive outcomes. The status of reproductive outcomes were assessed by the data collectors as a bad reproductive outcome if health care personnel experienced one of the following in their life event; inability to conceive (infertility), spontaneous abortion, congenital anomaly, delay conception, and premature death of an offspring (stillbirth). The questionnaires include, socio-demographic characteristics; reproductive history; history of anesthetics exposure, and history of substance use were prepared in English and translated into the local language, Afan Oromo and Amharic. To check for 
consistency, the questionnaires were further translated from Afan Oromo and Amharic to English by another person and the questionnaires were Self-administered.

Four trained diploma nurses who can speak both Afan oromo and Amharic were recruited from the health center and supervised by two BSc Nurse. One supervisor per 2 data collectors was assigned from the catchment health center.

Data collectors and supervisors were provided one-day training about the objective, the process of data collection, and field ethics and informed how to protect themselves and participants from covid-19. Each questionnaire was checked daily by the supervisors and the principal investigator.

A Pre-test was done in a non-study Hospital taking $5 \%$ of the total sample size. Based on the pre-test result clarifications, and corrections were done on inconsistency, ambiguity, comprehension, and exhaustiveness questions. Problems encountered were discussed with data collectors and solved immediately.

\section{Data analysis}

Each questionnaire was coded and checked manually for completeness and consistency. Then, data were entered into Epidata 4.6.1 and exported to SPSS version 24 where recoding, categorizing, computing, counting, and other statistical analysis were done.

Descriptive and the summary statistic were carried out to describe study participants according to different characteristics. The binary logistic regression model was fitted to identify factors associated with reproductive outcome status. Three models were fitted independently; for the whole sampled health care personnel (292), for the exposed health care personnel (146), and unexposed health care personnel (146). The model fitness for each model was tested by Hosmer-Lemeshow goodness of fitness, and the results of the test showed that the models were fit for every three models ( $P$-value $>0.05)$.

To check an interaction or effect modification of the independent variables, multi- co-linearity of the independent variables was checked using the variance inflation factor. There is no multi-co- linearity among the independent variables $(\mathrm{VIF}<10)$.

Using chi-square and bivariate, associations between dependent and several independent variables were examined one by one and those variables with $p$-value $<0.25$ were entered to multivariable logistic regression. Multivariable logistic regression analysis was employed to identify factors independently associated with reproductive outcome status by controlling the effect of potential confounding variables. Odds ratio (OR) with $95 \% \mathrm{Cl}$ was computed to assess the level of association and statistical significance. Statistical significance was declared using a p-value less than 0.05 . The result of this study is described in texts, tables, and graphs.

\section{Operational definition}




\section{Bad reproductive outcome}

when health care personnel experience diverse endpoints as the inability to conceive (infertility), delay conception, spontaneous abortion, congenital anomaly, and the premature death of an offspring (stillbirth), and if one of these noticed it is considered as bad reproductive outcomes(21)

\section{Good reproductive outcome}

when health care personnel are free of adverse reproductive health effects

\section{Results}

\section{Socio-Demographic Characteristics of Respondents}

A total of 483 health care personnel exposed and unexposed to anesthesia were approached to participate in this study. Of this number, $292 \mathrm{HCPs}$ were reached for final analysis while 191 were excluded from the study. The mean age of the respondent was $32.95 \pm 6.46$ years and it ranges from 21 to 59 in both groups. The highest proportion of the respondents was found in the age group 30-39 years in both groups. The majority 97 of exposed health care personnel have 2-5year of service and unexposed health care personnel have 65 from 6-9 years of service. Among the eight health facilities, Jimma medical center (JMC) has high exposure status which accounts for $31.2 \%$. Nurses have the highest proportion among all professions in both groups as detailed in (Table 1). 
Table 1

Socio-demographic characteristics among HCPs in Jimma zone public hospital, southwest Ethiopia, 2021

\begin{tabular}{|c|c|c|c|c|c|}
\hline \multirow[t]{2}{*}{ Variable } & \multirow[t]{2}{*}{ Category } & \multicolumn{2}{|c|}{$\begin{array}{l}\text { Exposed } \\
\text { group }(n=146)\end{array}$} & \multicolumn{2}{|c|}{ Unexposed $(n=146)$} \\
\hline & & Frequency & Percent & Frequency & Percent \\
\hline \multirow[t]{4}{*}{ Age group } & $20-29$ & 46 & 15.8 & 46 & 15.8 \\
\hline & $30-39$ & 80 & 27.4 & 80 & 27.4 \\
\hline & $40-49$ & 15 & 5.1 & 15 & 5.1 \\
\hline & $>49$ & 5 & 1.7 & 5 & 1.7 \\
\hline \multirow[t]{2}{*}{ Sex } & Male & 89 & 30.5 & 89 & 30.5 \\
\hline & Female & 57 & 19.5 & 57 & 19.5 \\
\hline \multirow[t]{5}{*}{ Educational status } & Diploma & 16 & 5.5 & 16 & 5.5 \\
\hline & BSc & 75 & 25.7 & 75 & 25.7 \\
\hline & MSc & 26 & 8.9 & 26 & 8.9 \\
\hline & Specialty & 25 & 8.6 & 25 & 8.6 \\
\hline & Sub-specialty & 4 & 1.4 & 4 & 1.4 \\
\hline \multirow[t]{8}{*}{ Group of profession } & Anesthesia & 41 & 14 & 0 & 0 \\
\hline & Gyn/obs & 14 & 4.8 & 0 & 0 \\
\hline & Surgery & 10 & 3.4 & 0 & 0 \\
\hline & OMF/ENT & 1 & 0.35 & 0 & 0 \\
\hline & Ophthalmology & 7 & 2.4 & 0 & 0 \\
\hline & Nurse & 73 & 25 & 109 & 37.3 \\
\hline & Medicine & 0 & 0 & 18 & 6.2 \\
\hline & Pediatrics & 0 & 0 & 19 & 6.5 \\
\hline \multirow[t]{4}{*}{ Place of work } & OR & 138 & 47.3 & 0 & 0 \\
\hline & PACU & 2 & 0.7 & 0 & 0 \\
\hline & ICU & 6 & 2.1 & 0 & 0 \\
\hline & Other wards & 0 & 0 & 146 & 50 \\
\hline
\end{tabular}




\begin{tabular}{|c|c|c|c|c|c|}
\hline \multirow[t]{2}{*}{ Variable } & \multirow[t]{2}{*}{ Category } & \multicolumn{2}{|c|}{$\begin{array}{l}\text { Exposed } \\
\text { group }(n=146)\end{array}$} & \multicolumn{2}{|c|}{ Unexposed $(n=146)$} \\
\hline & & Frequency & Percent & Frequency & Percent \\
\hline \multirow[t]{4}{*}{ Year of service } & 2-5 year & 97 & 32.2 & 46 & 17.5 \\
\hline & 6-9 year & 36 & 12.3 & 65 & 22.3 \\
\hline & 10-15 year & 11 & 3.8 & 33 & 11.3 \\
\hline & $>15$ & 2 & 0.7 & 2 & 0.7 \\
\hline \multirow[t]{2}{*}{ Married/couple when } & For two years & 19 & 6.5 & 31 & 10.6 \\
\hline & Before two years & 127 & 45.5 & 115 & 39.4 \\
\hline \multirow{3}{*}{$\begin{array}{l}\text { If married/coupled } \\
\text { when }\end{array}$} & Before service & 57 & 19.5 & 7 & 2.4 \\
\hline & At the start of service & 36 & 12.3 & 33 & 11.3 \\
\hline & After service & 53 & 18.2 & 106 & 36.3 \\
\hline \multirow[t]{3}{*}{ couple's age } & $19-29$ & 70 & 24 & 56 & 19.2 \\
\hline & $30-40$ & 66 & 22.6 & 74 & 25.3 \\
\hline & $\geq 41$ & 10 & 3.4 & 16 & 5.5 \\
\hline \multirow[t]{3}{*}{ Couple's job } & Exposed HCP & 11 & 3.8 & 5 & 1.7 \\
\hline & Un exposed HCP & 50 & 17.1 & 49 & 16.8 \\
\hline & NonHCP & 85 & 29.1 & 92 & 31.55 \\
\hline \multirow[t]{8}{*}{ Health Facility } & Jimma medical center & 91 & 31.2 & 91 & 31.5 \\
\hline & Shenan Gibe Hospital & 15 & 5.1 & 15 & 5.1 \\
\hline & Agaro Hospital & 11 & 3.8 & 11 & 3.8 \\
\hline & $\begin{array}{l}\text { Seka chokorsa } \\
\text { hospital }\end{array}$ & 6 & 2.1 & 6 & 2.1 \\
\hline & Dedo Hosnital & 7 & 2.4 & 7 & 2.4 \\
\hline & immu Genet Hesnital & 7 & 2.4 & 7 & 2.4 \\
\hline & Omo Neda Hosnital & 5 & 1.7 & 4 & 1.4 \\
\hline & Setama Hospital & 5 & 1.7 & 4 & 1.4 \\
\hline
\end{tabular}

Reproductive history of health care personnel 
The majority 272 (93.2\%) of the respondent's couples experienced at least one and above pregnancy. The average number of pregnancies per woman was $1.23 \pm 0.46$ for exposed HCPs and $1.27 \pm 0.45$ for unexposed HCPs.

Thirty (10.3\%) of the respondents among exposed had experienced spontaneous abortion, of which eight (2.7\%) experienced more than one spontaneous abortion after this occupational exposure. The percentage of children per exposed HCPs was (46.6\%) which is slightly less than the percentages of unexposed HCPs (51.4\%). Of the participants, $29.8 \%$ among exposed and $37.2 \%$ among unexposed conceived in $\leq 6$ months, $14.3 \%$ and $12.2 \%$ in $6-12$ months, and $4.4 \%$ and $2.2 \%$ exposed to and unexposed conceived after 1 year consequently. The proportion of being delivered only female child $46(15.8 \%)$ among exposed was higher than that of unexposed 26 (8.9\%). A high percentage of inability to conceive was observed among exposed health care personnel 14(4.8\%) as compared with unexposed health care personnel $6(2.1 \%)$. The male to female ratio among exposed was 0.8 while among unexposed was 2.03 (Table 2). 
Table 2

Reproductive history of health care personnel in Jimma zone public hospitals, southwest, Ethiopia, 2021

\begin{tabular}{|c|c|c|c|c|c|}
\hline \multirow[t]{2}{*}{ Variable } & \multirow[t]{2}{*}{ Category } & \multicolumn{2}{|c|}{ Exposed $(n=146$} & \multicolumn{2}{|c|}{ Unexposed $(n=146)$} \\
\hline & & Frequency & Percent & Frequency & Percent \\
\hline \multirow[t]{2}{*}{ contraceptive use } & Yes & 109 & 37.3 & 37 & 12.7 \\
\hline & No & 112 & 38.4 & 34 & 11.6 \\
\hline \multirow[t]{4}{*}{ Type of contraceptives } & OCP & 4 & 1.8 & 7 & 5 \\
\hline & Dipo & 46 & 20.8 & 57 & 46.6 \\
\hline & Implanol & 54 & 24.4 & 44 & 44.3 \\
\hline & IUD & 5 & 2.3 & 4 & 4.1 \\
\hline \multirow[t]{2}{*}{ Fertility status } & Conceived & 132 & 45.25 & 140 & 47.9 \\
\hline & Unable conceive & 14 & 4.8 & 6 & 2.1 \\
\hline \multirow[t]{4}{*}{ Duration of conception } & Within a month & 43 & 15.8 & 60 & 22.1 \\
\hline & Withinsix month & 38 & 14 & 41 & 15.1 \\
\hline & Within a year & 39 & 14.3 & 33 & 12.1 \\
\hline & Above one year & 16 & 4.4 & 8 & 2.2 \\
\hline Delivered normal live child & Yes & 125 & 47.7 & 133 & 50.8 \\
\hline \multirow[t]{2}{*}{ Delivered preterm } & Yes & 8 & 3.1 & 0 & 0 \\
\hline & No & 115 & 41.1 & 132 & 51.1 \\
\hline \multirow[t]{2}{*}{ spontaneous abortion } & Yes & 30 & 10.3 & 19 & 6.5 \\
\hline & No & 96 & 37.1 & 114 & 44 \\
\hline \multirow[t]{2}{*}{ Delivered congenital anomaly } & Yes & 5 & 2 & 0 & 0 \\
\hline & No & 119 & 46.5 & 132 & 45.2 \\
\hline \multirow[t]{2}{*}{ Still birth } & Yes & 4 & 1.6 & 3 & 1 \\
\hline & No & 120 & 46.9 & 129 & 44.2 \\
\hline Total number of child & & 126 & 46.6 & 133 & 45.5 \\
\hline \multirow[t]{2}{*}{ Sex ratio } & Male & $37 / 46$ & 0.80 & $53 / 26$ & \\
\hline & Female & & & 2.03 & \\
\hline
\end{tabular}

\section{History of substance use}


Among the total participants, the majority (19.9\%) in exposed HCPs and $26.7 \%$ in unexposed HCPs use a substance. 47 (34.6\%) exposed HCPs and 64 (47.1\%) unexposed HCPs use alcohol. The list used substances in two groups was shisha (Table 3 ).

Table 3

History of substance use among HCPs in Jimma zone public hospital, southwest Ethiopia, 2021

\begin{tabular}{|llllll|}
\hline Variable & Response & \multicolumn{2}{c}{ Exposed $(\mathbf{n}=\mathbf{1 4 6})$} & Un Exposed $(\mathbf{n}=146)$ \\
\cline { 3 - 6 } & & Frequency & percent & & Percent \\
\hline Substance use & Yes & 58 & 19.9 & 78 & 26.7 \\
& No & 88 & 30.1 & 68 & 23.3 \\
\hline Hx of alcohol use & Yes & 47 & 34.6 & 64 & 21.9 \\
& No & 11 & 8.1 & 14 & 4.8 \\
Hx of cigarettes smoking & Yes & 12 & 8.8 & 14 & 4.8 \\
& No & 46 & 33.8 & 64 & 21.9 \\
\hline Hx of shisha use & Yes & 2 & 1.5 & 3 & 1 \\
& No & 56 & 41.2 & 75 & 25.7 \\
Hx of khat use & Yes & 29 & 21.3 & 38 & 13 \\
& No & 29 & 21.3 & 40 & 13.7 \\
\hline
\end{tabular}

\section{The overall magnitude of reproductive outcomes}

The overall magnitude of bad reproductive outcomes among health care personnel in Jimma zone public hospital was $\mathbf{3 0 . 8 \%}$, of which spontaneous abortion accounted for more than half of the rest components of reproductive outcomes $16.8 \%$. The comparison of reproductive outcome status component among exposed and unexposed HCPs was calculated separately (Figure 2).

The overall magnitude of bad reproductive outcome status among health care personnel between a group and within a group as shown below and was higher among exposed health care personnel (as shown Figure 3).

\section{Predictors of reproductive outcome status}

Binary logistic regressions were applied to identify associated independent variables. To control the effect of confounders, a multiple logistic regression was done. The factors included in the model were 
those that showed association at the binary logistic regression analysis at a cut-off value (value $\leq 0.25$ ) and then those in multivariable logistic regression with value $<0.05$ were statically significant

\section{Overall Factors Associated with reproductive outcomes status among health care personnel $(n=292)$}

Bivariate analysis was done among the whole sample and ten variables met the criteria for multivariable logistic regression.

In multivariate analysis, the exposure status, cigarette smoking and Depo-Provera users were independently associated with the effect of anesthetics exposure on reproductive outcomes. Accordingly, health care personnel who were exposed to anesthesia were $\mathbf{3 . 1 7}$ times more likely to develop bad reproductive outcome as compared with those who were not exposed (AOR $=3.1795 \% \mathrm{Cl}$; 1.40-7.19) $\mathrm{P}<0.006$; health care personnel who smoke cigarettes were $\mathbf{8 . 4 4}$ times more likely to develop bad reproductive outcomes than those who were not smoke cigarettes (AOR=8.44, 95\% Cl; 1.93-36.91) $\mathrm{P}<0.005$ and Depo-Provera users were 6 times more likely to develop bad reproductive outcome if compared to other contraceptive users(AOR=6.01 95\% Cl:0.45-79.26) $\mathrm{P}<0.04$ (Table 4). 
Table 4

Logistic regression of factors associated with reproductive outcome status among health care personnel in Jimma zone public hospitals, southwest Ethiopia, 2021

\begin{tabular}{|c|c|c|c|c|c|c|c|}
\hline \multirow[t]{2}{*}{ Variables } & \multirow[t]{2}{*}{ Category } & \multicolumn{2}{|c|}{$\begin{array}{l}\text { Dependent } \\
\text { variable }\end{array}$} & \multicolumn{2}{|c|}{ Bivariate result } & \multicolumn{2}{|c|}{ Multivariate result } \\
\hline & & Bad & Good & $\begin{array}{l}\text { COR } \\
(95 \% \mathrm{Cl})\end{array}$ & P-V & $\mathrm{AOR}(95 \% \mathrm{Cl})$ & P-V \\
\hline \multirow[t]{2}{*}{ Exposure status } & Exposed & 53 & 93 & $\begin{array}{l}2.51(1.46- \\
4.29)\end{array}$ & 0.002 & $\begin{array}{l}3.17(1.40- \\
7.19)\end{array}$ & $0.006 *$ \\
\hline & Unexposed & 27 & 119 & 1 & & & 1 \\
\hline \multirow[t]{4}{*}{ Age } & $20-29$ & 23 & 73 & 1 & & & 1 \\
\hline & $30-39$ & 46 & 123 & $\begin{array}{l}0.66(0.67- \\
2.11)\end{array}$ & 0.56 & $\begin{array}{l}0.84(0.12- \\
5.61)\end{array}$ & 0.86 \\
\hline & $40-49$ & 8 & 14 & $\begin{array}{l}0.67(0.67- \\
4.86)\end{array}$ & 0.23 & $\begin{array}{l}0.28(0.01- \\
21.14)\end{array}$ & 0.56 \\
\hline & $>49$ & 3 & 2 & $\begin{array}{l}0.74(0.05- \\
2.78)\end{array}$ & 0.09 & & 1.0 \\
\hline \multirow[t]{5}{*}{$\begin{array}{l}\text { Educational } \\
\text { status }\end{array}$} & Diploma & 9 & 23 & $\begin{array}{l}0.23(0.46- \\
1.19)\end{array}$ & 0.08 & $\begin{array}{l}0.61(0.03- \\
11.93)\end{array}$ & 0.74 \\
\hline & BSc & 37 & 113 & $\begin{array}{l}0.19(0.04- \\
0.86)\end{array}$ & 0.03 & $\begin{array}{l}0.45(0.02- \\
7.69)\end{array}$ & 0.58 \\
\hline & MSc & 17 & 35 & $\begin{array}{l}0.42(0.08- \\
2.02)\end{array}$ & 0.28 & $\begin{array}{l}0.75(0.05- \\
11.09)\end{array}$ & 0.83 \\
\hline & Specialty & 12 & 38 & $\begin{array}{l}0.17(0.03- \\
0.84)\end{array}$ & 0.03 & $\begin{array}{l}0.09(0.01- \\
0.92)\end{array}$ & 0.06 \\
\hline & $\begin{array}{l}\text { Sub } \\
\text { specialty }\end{array}$ & 5 & 3 & 1 & & & \\
\hline \multirow[t]{4}{*}{ Year of service } & $2-5$ & 37 & 106 & 1 & & & 1 \\
\hline & $6-9$ & 27 & 74 & $\begin{array}{l}0.97(0.34- \\
2.74)\end{array}$ & 0.95 & $\begin{array}{l}1.27(0.02- \\
74.75)\end{array}$ & 0.90 \\
\hline & $10-15$ & 15 & 29 & $\begin{array}{l}1.37(0.47- \\
4.25)\end{array}$ & 0.57 & $\begin{array}{l}0.59(0.07- \\
49.48)\end{array}$ & 0.81 \\
\hline & $>15$ & 1 & 3 & $\begin{array}{l}0.88(0.07- \\
10.3)\end{array}$ & 0.92 & $\begin{array}{l}1.03(0.07- \\
156.43)\end{array}$ & \\
\hline \multirow[t]{2}{*}{$\begin{array}{l}\text { Married/coupled } \\
\text { when }\end{array}$} & $\begin{array}{l}\text { For two } \\
\text { years }\end{array}$ & 8 & 42 & 1 & & & \\
\hline & $\begin{array}{l}\text { Before } \\
\text { 2years }\end{array}$ & 72 & 170 & $\begin{array}{l}2.22(0.99- \\
4.97)\end{array}$ & 0.05 & $\begin{array}{l}2.0(0.62- \\
6.49)\end{array}$ & 0.24 \\
\hline When married & Before & 23 & 41 & 1 & & & 1 \\
\hline
\end{tabular}




\begin{tabular}{|c|c|c|c|c|c|c|c|}
\hline & \multicolumn{7}{|l|}{ service } \\
\hline & $\begin{array}{l}\text { At start of } \\
\text { service }\end{array}$ & 22 & 47 & $\begin{array}{l}0.83(0.40- \\
171)\end{array}$ & 0.62 & $\begin{array}{l}0.91(0.18- \\
4.58)\end{array}$ & 0.91 \\
\hline & After service & 35 & 124 & $\begin{array}{l}0.53(0.26- \\
0.94)\end{array}$ & 0.03 & $\begin{array}{l}0.27(0.05- \\
1.27)\end{array}$ & 0.09 \\
\hline \multirow[t]{3}{*}{ Couple job } & $\begin{array}{l}\text { Exposed } \\
\text { HCP }\end{array}$ & 5 & 11 & $\begin{array}{l}1.46(0.48- \\
4.44)\end{array}$ & 0.50 & $\begin{array}{l}1.95(0.03- \\
124.51)\end{array}$ & 0.75 \\
\hline & $\begin{array}{l}\text { Unexposed } \\
\text { HCP }\end{array}$ & 33 & 66 & $\begin{array}{l}1.60(0.93- \\
2.76)\end{array}$ & 0.08 & $\begin{array}{l}0.78(0.16- \\
3.71)\end{array}$ & 0.76 \\
\hline & Non HCP & 42 & 135 & 1 & & & 1 \\
\hline \multirow[t]{4}{*}{$\begin{array}{l}\text { Type of } \\
\text { contraceptives }\end{array}$} & OCP & 4 & 7 & $\begin{array}{l}4.57(0.40- \\
51.1)\end{array}$ & 0.21 & $\begin{array}{l}13.92(0.78- \\
47.6)\end{array}$ & 0.17 \\
\hline & Dipo & 23 & 80 & $\begin{array}{l}2.30(0.27- \\
19.3)\end{array}$ & 0.4 & $\begin{array}{l}6.01(0.45- \\
79.26)\end{array}$ & $0.04 *$ \\
\hline & Implanol & 30 & 68 & $\begin{array}{l}3.52(0.42- \\
29.4)\end{array}$ & 0.24 & $\begin{array}{l}6.41(0.49- \\
83.23)\end{array}$ & 0.15 \\
\hline & IUD & 1 & 8 & 1 & & & 1 \\
\hline \multirow[t]{2}{*}{ Use alcohol } & Yes & 46 & 65 & $\begin{array}{l}1.82(0.70- \\
4.71)\end{array}$ & 0.21 & $\begin{array}{l}0.67(0.06- \\
7.24)\end{array}$ & 0.74 \\
\hline & No & 7 & 18 & 1 & & & 1 \\
\hline \multirow[t]{2}{*}{$\begin{array}{l}\text { Smoke } \\
\text { cigarattes }\end{array}$} & Yes & 19 & 7 & $\begin{array}{l}6.06(2.33- \\
15.7)\end{array}$ & 0.01 & $\begin{array}{l}8.44(1.93- \\
36.91)\end{array}$ & $0.005^{\star}$ \\
\hline & No & 34 & 76 & 1 & & 1 & \\
\hline
\end{tabular}

\section{Factors Associated with reproductive outcome status among exposed health care personnel}

Bivariate analysis was carried out and twelve variables were associated with reproductive outcomes among exposed health care personnel. In multivariable analysis, three of them were found to be significantly associated. It was found in this study that the exposed couple HCPs were nearly 2 times more likely to develop bad reproductive outcomes if compared with the unexposed couple health care personnel (AOR= $1.8495 \%, \mathrm{Cl} 0.46-7.01) \mathrm{P}<0.03$ : Health care personnel who had $30-45 \mathrm{~h} / \mathrm{w}$ exposure were nearly 4 times more likely to develop bad reproductive outcome as compared with those who spent less hour per week in the potential area of exposure(AOR=11.94,95\% Cl:1.25-24.93) $\mathrm{P}<0.03$. Anesthesia personnel was 2 times more likely to develop bad reproductive outcomes when compared with other exposed health care personnel (AOR=2 95\%, Cl: 0.09-9.13) $\mathrm{P}<0.042$ (Table 5). 
Table 5

Logistic regression analysis among exposed health care personnel in Jimma zone public hospitals, southwest Ethiopia, 2021 $(n=146)$

\begin{tabular}{|c|c|c|c|c|c|c|c|}
\hline \multirow[t]{2}{*}{ Variable } & \multirow[t]{2}{*}{ Category } & \multicolumn{2}{|c|}{$\begin{array}{l}\text { Reproductive } \\
\text { outcome }\end{array}$} & \multicolumn{2}{|c|}{ Bivariate result } & \multicolumn{2}{|l|}{ Multivariate result } \\
\hline & & Bad & Good & $\operatorname{COR}(95 \% \mathrm{Cl})$ & PV & AOR $(95 \% \mathrm{Cl})$ & $\begin{array}{l}\mathrm{P} \text { - } \\
\text { value }\end{array}$ \\
\hline \multirow[t]{2}{*}{ Sex } & Male & 28 & 61 & 1 & & & \\
\hline & Female & 25 & 32 & $\begin{array}{l}1.70(0.85- \\
3.38)\end{array}$ & 0.13 & $3.17(1.12-8.96)$ & 0.21 \\
\hline \multirow[t]{5}{*}{$\begin{array}{l}\text { Educational } \\
\text { status }\end{array}$} & Diploma & 7 & 9 & $\begin{array}{l}0.58(0.09- \\
3.50)\end{array}$ & 0.55 & 1.82(0.12-27.79) & 0.66 \\
\hline & BSc & 21 & 39 & $\begin{array}{l}0.40(0.08- \\
1.97)\end{array}$ & 0.26 & 1.34(0.09-18.49) & 0.82 \\
\hline & MSc & 13 & 16 & $\begin{array}{l}0.60(0.11- \\
3.22)\end{array}$ & 0.56 & $0.81(0.09-8.80)$ & 0.93 \\
\hline & Specialty & 8 & 26 & $\begin{array}{l}0.23(0.04- \\
1.25)\end{array}$ & 0.09 & $0.47(0.05-4.26)$ & 0.50 \\
\hline & Sub specialty & 4 & 3 & 1 & & 1 & 1 \\
\hline \multirow[t]{5}{*}{ Profession } & Anesthesia & 15 & 26 & $\begin{array}{l}2.01(0.37- \\
10.9)\end{array}$ & 0.21 & $2(0.09-9.13)$ & $0.04 *$ \\
\hline & Gyn/obs & 11 & 11 & $\begin{array}{l}3.50(0.59- \\
20.7)\end{array}$ & 0.16 & $2.15(0.24-19.12)$ & 0.49 \\
\hline & Nurse & 19 & 37 & $\begin{array}{l}1.79(0.34- \\
9.50)\end{array}$ & 0.49 & $0.88(0.07-11.16)$ & 0.92 \\
\hline & Surgery & 6 & 11 & $\begin{array}{l}1.90(0.29- \\
12.2)\end{array}$ & 0.46 & $1.89(0.26-13.59)$ & 0.52 \\
\hline & Ophthalmology & 2 & 8 & 1 & & 1 & 1 \\
\hline \multirow{4}{*}{$\begin{array}{l}\text { Year of } \\
\text { service }\end{array}$} & $2-5$ & 30 & 67 & 1 & & 1 & 1 \\
\hline & $6-9$ & 16 & 20 & $\begin{array}{l}2.00(0.63- \\
6.33)\end{array}$ & 0.23 & $1.08(0.35-3.35)$ & 0.89 \\
\hline & $10-15$ & 6 & 5 & $\begin{array}{l}3.00(0.65- \\
13.6)\end{array}$ & 0.15 & $1.82(0.46-7.24)$ & 0.39 \\
\hline & $>15$ & 1 & 1 & $\begin{array}{l}2.50(0.13- \\
46.7)\end{array}$ & 0.54 & $2.60(38-17.68)$ & 0.32 \\
\hline \multirow[t]{2}{*}{$\begin{array}{l}\text { If married } \\
\text { when }\end{array}$} & Before service & 22 & 35 & $\begin{array}{l}0.95(0.44- \\
2.06)\end{array}$ & 0.91 & $0.61(0.23-1.63)$ & 0.32 \\
\hline & $\begin{array}{l}\text { At start of } \\
\text { service }\end{array}$ & 10 & 26 & $\begin{array}{l}0.58(0.23- \\
1.46)\end{array}$ & 0.25 & $1.0(0.44-2.26)$ & 0.99 \\
\hline
\end{tabular}




\begin{tabular}{|c|c|c|c|c|c|c|c|}
\hline & After service & 21 & 32 & 1 & & 1 & 1 \\
\hline \multirow[t]{3}{*}{ Couple job } & Exposed HCP & 5 & 6 & $\begin{array}{l}1.89(0.52- \\
6.75)\end{array}$ & 0.02 & $1.84(0.46-7.01)$ & $0.03 *$ \\
\hline & $\begin{array}{l}\text { Unexposed } \\
\text { HCP }\end{array}$ & 22 & 28 & $\begin{array}{l}1.78(0.86- \\
3.67)\end{array}$ & 0.11 & $1.70(0.80-3.63)$ & 0.16 \\
\hline & Non HCP & 26 & 59 & 1 & & 1 & 1 \\
\hline \multirow[t]{4}{*}{$\begin{array}{l}\text { Substance } \\
\text { use }\end{array}$} & Alcohol & 27 & 20 & $\begin{array}{l}2.36(0.60- \\
9.18)\end{array}$ & 0.21 & $4.29(0.50-36.36)$ & 0.18 \\
\hline & $\begin{array}{l}\text { Smoke } \\
\text { cigarettes }\end{array}$ & 22 & 24 & $\begin{array}{l}3.27(0.78- \\
13.6)\end{array}$ & 0.10 & $8.57(0.88-74.26)$ & 0.07 \\
\hline & Shisha use & 2 & 0 & & & -ב-ב-ב-ב-ב & \\
\hline & Chewing khat & 17 & 12 & 1 & 1 & 1 & \\
\hline \multirow[t]{3}{*}{$\begin{array}{l}\text { Inhalational } \\
\text { agent }\end{array}$} & Halothane & 50 & 88 & $\begin{array}{l}0.94(0.21- \\
4.13)\end{array}$ & 0.94 & $0.67(0.14-3.09)$ & 0.60 \\
\hline & Iso flurane & 19 & 54 & $\begin{array}{l}0.40(0.20- \\
0.81)\end{array}$ & 0.01 & $0.38(0.07-2.09)$ & 0.27 \\
\hline & Sevoflurane & 0 & 2 & 1 & & 1 & \\
\hline \multirow{4}{*}{$\begin{array}{l}\text { Service } \\
\text { exposure } \\
\text { h/wk }\end{array}$} & $<15$ & 2 & 1 & & & 1 & 1 \\
\hline & $15-30$ & 9 & 32 & $\begin{array}{l}2.38(0.01- \\
1.73)\end{array}$ & 0.49 & $12.17(1.47100 .18)$ & 0.12 \\
\hline & $31-45$ & 21 & 32 & $\begin{array}{l}0.33(0.02- \\
3.85)\end{array}$ & 0.02 & $3.94(1.25-24.93$ & $0.03^{*}$ \\
\hline & $>45$ & 21 & 25 & $\begin{array}{l}0.78(0.03- \\
4.96)\end{array}$ & 0.54 & $\longrightarrow$ & \\
\hline \multirow{3}{*}{$\begin{array}{l}\text { Status of } \\
\text { scavenging } \\
\text { system }\end{array}$} & Standardized & 4 & 21 & 1 & & & \\
\hline & Semi secured & 21 & 27 & $\begin{array}{l}4.08(1.21- \\
13.7)\end{array}$ & 0.02 & $5.07(0.55-46.59)$ & 0.15 \\
\hline & $\begin{array}{l}\text { Not secured } \\
\text { and leaks }\end{array}$ & 15 & 21 & $\begin{array}{l}3.75(1.06- \\
13.19)\end{array}$ & 0.03 & $1.20(0.10-13.42)$ & 0.87 \\
\hline
\end{tabular}

\section{Factors Associated with reproductive outcome status among unexposed health care personnel}

In the bivariate analysis, the factors associated with reproductive outcomes among unexposed health care personnel entered to multivariable model and fitted. Health care personnel who smoke cigarettes were 6.53 times more likely to develop bad reproductive outcome as compared to with those who didn't smoke cigarettes $(\mathrm{AOR}=6.53,95 \% \mathrm{Cl} 1.32-32.63) \mathrm{P}<0.02$ and health care personnel whose couple age is greater than 41 years were 5.87 times more likely to develop bad reproductive outcomes with those whose couple age is less than 40years (AOR=5.87, 95\% Cl: 1.56-22.06) $p<0.009$ (Table 6). 
Table 6

Logistic regression analysis among unexposed health care personnel in Jimma zone public hospitals, southwest Ethiopia, 2021 $(n=146)$

\begin{tabular}{|c|c|c|c|c|c|c|c|}
\hline \multirow[t]{2}{*}{ Variables } & \multirow[t]{2}{*}{ Category } & \multicolumn{2}{|c|}{$\begin{array}{l}\text { Reproductive } \\
\text { outcome }\end{array}$} & \multicolumn{2}{|l|}{ Bivariate result } & \multicolumn{2}{|c|}{ Multivariate result } \\
\hline & & Bad & Good & $\operatorname{coR}(95 \% \mathrm{Cl}$ & $P-V$ & AOR $(95 \% \mathrm{Cl})$ & $P-V$ \\
\hline \multirow[t]{4}{*}{ Age } & $20-29$ & 5 & 45 & 1 & & 1 & 1 \\
\hline & $30-39$ & 20 & 66 & $\begin{array}{l}2.77(0.97- \\
7.90)\end{array}$ & 0.05 & $\begin{array}{l}1.89(0.45- \\
7.94)\end{array}$ & 0.38 \\
\hline & $40-49$ & 1 & 6 & $\begin{array}{l}1.50(0.14- \\
15.10)\end{array}$ & 0.73 & $\begin{array}{l}0.76(0.04- \\
13.33)\end{array}$ & 0.85 \\
\hline & $>49$ & 1 & 2 & $\begin{array}{l}0.82(0.06- \\
67.12)\end{array}$ & 0.92 & $\begin{array}{l}1.02(0.09- \\
93.12)\end{array}$ & 0.90 \\
\hline \multirow[t]{2}{*}{ Sex } & Male & 22 & 67 & 1 & & & \\
\hline & Female & 5 & 52 & $\begin{array}{l}0.29(0.10- \\
0.82)\end{array}$ & 0.02 & $\begin{array}{l}5.76(1.14- \\
24.63)\end{array}$ & 0.07 \\
\hline \multirow[t]{2}{*}{$\begin{array}{l}\text { When } \\
\text { coupled }\end{array}$} & $\begin{array}{l}\text { For } 2 \\
\text { year }\end{array}$ & 3 & 28 & 1 & & 1 & 1 \\
\hline & $\geq 2$ year & 24 & 91 & $\begin{array}{l}0.46(0.68- \\
8.79)\end{array}$ & 0.16 & $\begin{array}{l}5.76(0,59- \\
56.28)\end{array}$ & 0.13 \\
\hline \multirow[t]{3}{*}{ Age of couple } & $19-29$ & 9 & 47 & 1 & & 1 & 1 \\
\hline & $30-40$ & 14 & 60 & $\begin{array}{l}1.21(0.48- \\
3.05)\end{array}$ & 0.67 & & \\
\hline & $\geq 41$ & 4 & 12 & $\begin{array}{l}1.74(0.45- \\
6.63)\end{array}$ & 0.41 & $\begin{array}{l}5.87(1.56- \\
22.06)\end{array}$ & $0.009 *$ \\
\hline \multirow[t]{2}{*}{$\begin{array}{l}\text { Smoke } \\
\text { cigarettes }\end{array}$} & Yes & 10 & 4 & $\begin{array}{l}10.83(2.89- \\
40.49)\end{array}$ & 0.000 & $\begin{array}{l}6.53(1.32- \\
32.31)\end{array}$ & $0.02 *$ \\
\hline & No & 12 & 52 & 1 & & & \\
\hline \multirow[t]{2}{*}{ Chewing khat } & Yes & 8 & 30 & $0.49(0.17-1.36$ & 0.17 & $\begin{array}{l}0.45(0.12- \\
1,66)\end{array}$ & 0.23 \\
\hline & No & 14 & 26 & 1 & & & \\
\hline
\end{tabular}

\section{Discussion}

This study revealed the effect of inhaled anesthetics exposure and its predictors on reproductive outcomes among exposed and unexposed health care personnel in Jimma zone public hospitals. 
Accordingly, the overall prevalence of bad reproductive outcomes was $30.8 \%$. The exposure type-specific prevalence of bad reproductive outcomes was $20.9 \% \%$ among exposed and $9.9 \%$ among unexposed health care personnel which shows there was a significant difference in the prevalence of bad reproductive outcomes among exposed health care personnel and unexposed health care personnel. The possible explanation for this variation may be that exposure to inhalational anesthetics may affect the reproductive outcome of exposed health care personnel compared to unexposed health care personnel. The overall prevalence of reproductive status in this study was higher as compared with the findings from a study conducted in Indonesia and Canada; the overall prevalence of reproductive status among exposed and unexposed health care personnel was $21 \%(1)$ and $18.7 \%$ respectively(14). This national discrepancy might be due to working environment, duration of exposure variation among the study hospitals. It also might be due to Variation of anesthesia machine status, quality of scavenging system, and operation room air conditioning, use of protective equipment and awareness of health care personnel to protect themselves, and the introduction of newer inhalational agents and changing anesthetic practice habits.

The overall prevalence in this study was lower than the studies conducted in Khartoum (47.2\%)(13), Egypt(32.5\%)(22), and Finland (33.1\%)(7). The difference might be due to the study design variation in which the cohort and experimental study design more investigate the problem intensively than comparative cross-sectional study.

Health care personnel that were exposed to inhalational anesthetics were associated with bad reproductive outcomes $(A O R=3.1795 \% \mathrm{Cl} ; 1.40-7.19) \mathrm{P}<0.006$. This finding was consistent with a study conducted in California $\mathrm{P}<0.007(23)$ and in London $\mathrm{P}<0.02$ (12). A meta-analysis shows that the absence of scavenging systems reported increased risks for spontaneous abortion as a consequence of increased anesthetics exposure (17). A study done in the Netherlands suggests reproductive toxicity has been the most persistent area of concern associated with occupational exposure to inhalational anesthetics. Ended the incidence of preterm delivery, congenital abnormalities and an increased abortion rate in females exposed to waste anesthetics were high compared with unexposed groups(9).

The cohort study done at zagazig university (AOR=1.49,95\% Cl:1.04-2.13) (24) and in turkey indicate that the incidence of abortion among female anesthesiologists, hospital operating room nurses, and wives of male anesthesiologists is greater than those for the normal population(25). This Increase in infertility, spontaneous abortion, and the risk of congenital anomalies among health personnel working in the operating room, suggests that anesthetics may harm the germ cells and lead to changes in sperm morphology and motility.

Another independently associated factor among the whole and unexposed health care personnel showed smoking cigarettes were 8.44 times more likely to develop bad reproductive outcomes compared to those who didn't smoke cigarettes (AOR=6.53,95\% Cl: 1.32-32.31). This study is nearly similar to a study done in France (AOR=7, 95\% Cl $2.13-7.65)$ (26). A meta-analysis of more than 2500 men from five separate 
studies revealed a significant decrease in sperm concentrations of current smokers compared with those who had never smoked(18).

This might be due to the effects of cigarette smoke being dose-dependent and are influenced by the presence of other toxic substances and hormonal status. Individual sensitivity, dose, time, and type of exposure also play a role in the impact of smoke constituents on human reproductive outcomes.

Depo-Provera was one of the associative risk factors for the bad reproductive outcome when compared with other contraceptive users (AOR=6.01 95\% Cl: 0.45-79.26) $\mathrm{P}<0.04$. This study is slightly higher than the study done in the UK(27)p $<0.01$. This discrepancy might be the study setting difference and the risk of these hormonal contraceptive were due to the increases in the viscosity of cervical mucus, thus creating a sticky barrier to spermatozoa and making the endometrium (the lining of the uterus) less suitable for implantation.

Being anesthesia professionals were associated with bad reproductive outcomes if compared with other health care professionals ( $\mathrm{AOR}=2.2295 \% \mathrm{Cl}: 0.10-7.01) \mathrm{P}<0.03$. This study is higher than the study done at the University of Massachusetts medical school (AOR=2 95\% Cl:0.8-13.24)(28). This is might be due to the anesthesia professionals being close exposed than any other exposed health care personnel, the difference might be due to the study setting and the inhalational agents

Health care personnel who were exposed to inhalation anesthetics for 30-45 h/W were nearly 4 times more likely to develop bad reproductive than those who have lower frequency exposure among exposed $(A O R=11.94,95 \% \mathrm{Cl}: 1.25-24.93) \mathrm{P}<0.03$. This study is higher than the study done in the Netherlands(9) and Australia (12). The difference might be due to the shortage of health care personnel manpower and this increase the long hour working in the area of anesthetic exposure in the study setting.

Other associated factors of bad reproductive outcomes among exposed health care personnel were being a couple of exposed health care personnel. This finding is in line with the study conducted in the U.S.A(29) and in India (30). Health care personnel whose paternal age is greater than 41 years were more likely to develop bad reproductive outcomes when compared with health care professionals whose age is less than 40 years. This study is similar to the study done in Canada (15) which suggests, aging has a negative consequence on reproductive outcomes. Another study done in Singapore and Israel supports that paternal age greater than 40 years was a risk factor for bad reproductive outcomes. This is due to the decreased number of oocytes as well as oocyte quality, decrease number and quality of semen, giving rise to lower fertilization and implantation rates, with fewer cycles reaching embryo transfer(27,31).

\section{Conclusion}

The Prevalence of bad reproductive outcomes was higher among exposed health care personnel if compared with unexposed health care personnel. Among the components of bad reproductive outcomes, spontaneous abortions were the dominant bad reproductive outcome experienced among exposed 
respondents and the delay of conception was 2 times fold increase among the exposed. The male to female ratio is low among exposed group.

\section{Abbreviations}

ACGIH

American Conference of Governmental Industrial Hygienists

AOR

adjusted Odd ratio

COR

crude odd ratio

ETT

Endotracheal tube

HCPs

Health care personnel

ICU

Intensive care unit

IAG

Inhalational anesthetic gas

JMC

Jimma Medical Center

$\mathrm{NIOSH}$

National Institute for Occupational Safety and Health

$\mathrm{N} 2 \mathrm{O}$

Nitrous oxide

OR

Operation room

PACU

Post anesthesia care unit

PPE

personnel protective equipment's

SPSS

static package for social science

WAG

waste anesthetics gases

WHO

world health organization

VIF

Variable inflation factor 


\section{Declarations}

\section{Ethics approval and consent to participate}

Ethical clearance was obtained from Jimma University Ethical Review Board. The study was also maintained the protocol of Helsinki declaration. A letter of permission was obtained from Jimma medical center and Jimma zone health office for all hospitals in the zone. Informed consent was taken from each participant and confidentiality was maintained by excluding the name of participants from the questionnaire and keeping the privacy of individual participants.

\section{Consent for publication}

Not Applicable

\section{Availability of data and materials}

The datasets used during the current study were available by requesting the corresponding author for reasonable quest.

\section{Competing Interests}

The authors declared that there is no competing interests.

\section{Funding}

The study was funded by Jimma University, postgraduate program for data collectors (IRB). The funder has no role in the design of the study and collection, analysis, and interpretation of data and in writing the manuscript.

\section{Authors' contributions}

MK, WR, and ZM participated in the conception of the study idea, designing, data analysis, interpretation of the findings, and write-up of the findings. TT, MB, MB, and GT are involved in designing methods, analysis, interpretation, and drafting of the manuscript.

\section{Acknowledgment}


Our greatest thanks were to Jimma medical center and all public hospitals in the Jimma zone for their cooperation and willingness through the process of the study. Again, we would like to express our deepest gratitude to the study participants for giving the necessary information.

\section{References}

1. Handayani R, Abdullah T, Naiem F, Mallongi A. Effects of Isoflurane Exposure to Fertility through Estrogen Gene Expression Effects of Isoflurane Exposure to Fertility through Estrogen Gene Expression in Operating Room Nurses. 2018;(January):10-7.

2. Anesthetists N, Assistants A. HHS Public Access. 2017;13(10):782-93.

3. Al-Ashour IAK, Abd-ali DK, Fallah MA, Kteo IQ. Effect Of Inhaled Anesthetics Gases On Health Staff Health Status In Al-Najaf City. 2014;3(12):383-8.

4. Kim S, Buro K, Tsui B. Elevated Waste Anesthetic Gas Concentration in the Paediatric Post- Anaesthesia Care Unit. 2018;46(5):362-7.

5. Casale T, Caciari T, Valeria M, Agostino P, Pia M, Capozzella A, et al. Anesthetic gases and occupationally exposed workers. Environ Toxicol Pharmacol [Internet]. 2013;37(1):267-74. Available from: http://dx.doi.org/10.1016/j.etap.2013.12.003

6. Chandrasekhar M, Rekhadevi P V, Sailaja N, Rahman MF, Reddy JP, Mahboob M, et al. Evaluation of genetic damage in operating room personnel exposed to anaesthetic gases. 2006;21(4):249-54.

7. Hemminki K, Kyyronen P, Lindbohm M. Spontaneous abortions and malformations in the offspring of nurses exposed to anaesthetic gases, cytostatic drugs, and other potential hazards in hospitals, based on registered information of outcome. 1985;141-7.

8. Smith JC, Bolon B. Atmospheric Waste Isoflurane Concentrations Using Conventional Equipment and Rat Anesthesia. 2002;41(2).

9. Burm AGL. Occupational hazards of inhalational anaesthetics. 2003;17(1):147-61.

10. Clifford T. Environmental Hazards: Waste Anesthesia Gases. J perianesthesia Nurs [Internet].2014;29(4):330-1.Available from: http://dx.doi.org/10.1016/j.jopan.2014.05.007

11. Hai-Xin Hua et al. Cellular and molecular mechanisms of oxidative stress in nerve injury, 2021.

12. Shirangi A. Associations of Unscavenged Anesthetic Gases and Long Working Hours With Preterm Delivery in Female Veterinarians. 2009;113(5):1008-17.

13. Mohammed. Occupational Hazards Associated with Exposure to Anesthetic Gases on Reproductive Health in Operating Theatre Staff in Three Hospitals in Khartoum State, Sudan. 2016 
14. Shuhaiber S, Einarson A, Radde IC, Sarkar M, Koren G. A PROSPECTIVE-controlled study of pregnant veterinary staff exposed to inhaled anesthetics and x-rays. 2002;15(4):363-73.

15. Guirguis SS, Pelmear PL, Roy ML, Wong L. Health effects associated with exposure to anaesthetic gases in Ontario hospital personnel. 1990;490-7.

16. Souza KM, Braz LG, Nogueira FR, Souza MB, Bincoleto LF, Aun AG, et al. Mutation Research / Fundamental and Molecular Mechanisms of Mutagenesis Occupational exposure to anesthetics leads to genomic instability , cytotoxicity and proliferative changes. Mutat Res - Fundam Mol Mech Mutagen [Internet]. 2016;791-792:42-8. Available from: http://dx.doi.org/10.1016/j.mrfmmm.2016.09.002

17. Services h. Waste anesthetic gases Occupational Hazards in Hospitals. 2018

18. Gabrielsen JS, Tanrikut C. Chronic exposures and male fertility : the impacts of environment, diet, and drug use on spermatogenesis. 2016;

19.RogérioC, OliveiraD. Exposição Ocupacional aResíduos deGases Anestésicos * Occupational Exposure to Anesthetic Gases Residue. 2009;59.

20. Risk FR.Guidelines for Reproductive Toxicity Risk Assessment.1996;61(October):56274-322.

21. Pike, Bethesda MD, adverse Reproductive outcomes in Families of Atomic Veterans,1995

22. Tankó B, Molnár L, Fülesdi B, Molnár C. Journal of Anesthesia \& Clinica I Occupational Hazards of Halogenated Volatile Anesthetics and their Prevention: Review of the Literature. 2014;5(7).

23. Sessler I, Michael J. Exposure of Postoperative Anesthetic Gases.2018;17(8):156-6

24. Ma H. Group of nurses exposed to anesthetic gases in operating rooms at zagazig university hospitals. 2018;42(1):105-22.

25. Ceyhan A, Cincik M, Bedir S, Ustun H, Dagli G, Kalender H. Archives of Andrology effects of exposure to new inhalational anesthetics on spermatogenesis and sperm morphology in rabbits. 2009;5016.

26. Dechanet C, Anahory T, Daude JCM, Quantin X, Reyftmann L, Hamamah S, et al. Effects of cigarette smoking on reproduction. 2011;17(1):76-95.

27.Wiener-megnazi Z, Auslender R, Dirnfeld M. Advanced paternal age and reproductive outcome. 2012; (May 2011):69-76.

28.Figa-talamancal.Reproductive Problems among Women Health Care Workers: Epidemiologic Evidence and Preventive Strategies. 2000;22(2).

29. Ravishankar M. Anaesthesia practice and reproductive outcomes: Facts unveiled. 2015; 


\section{Figures}

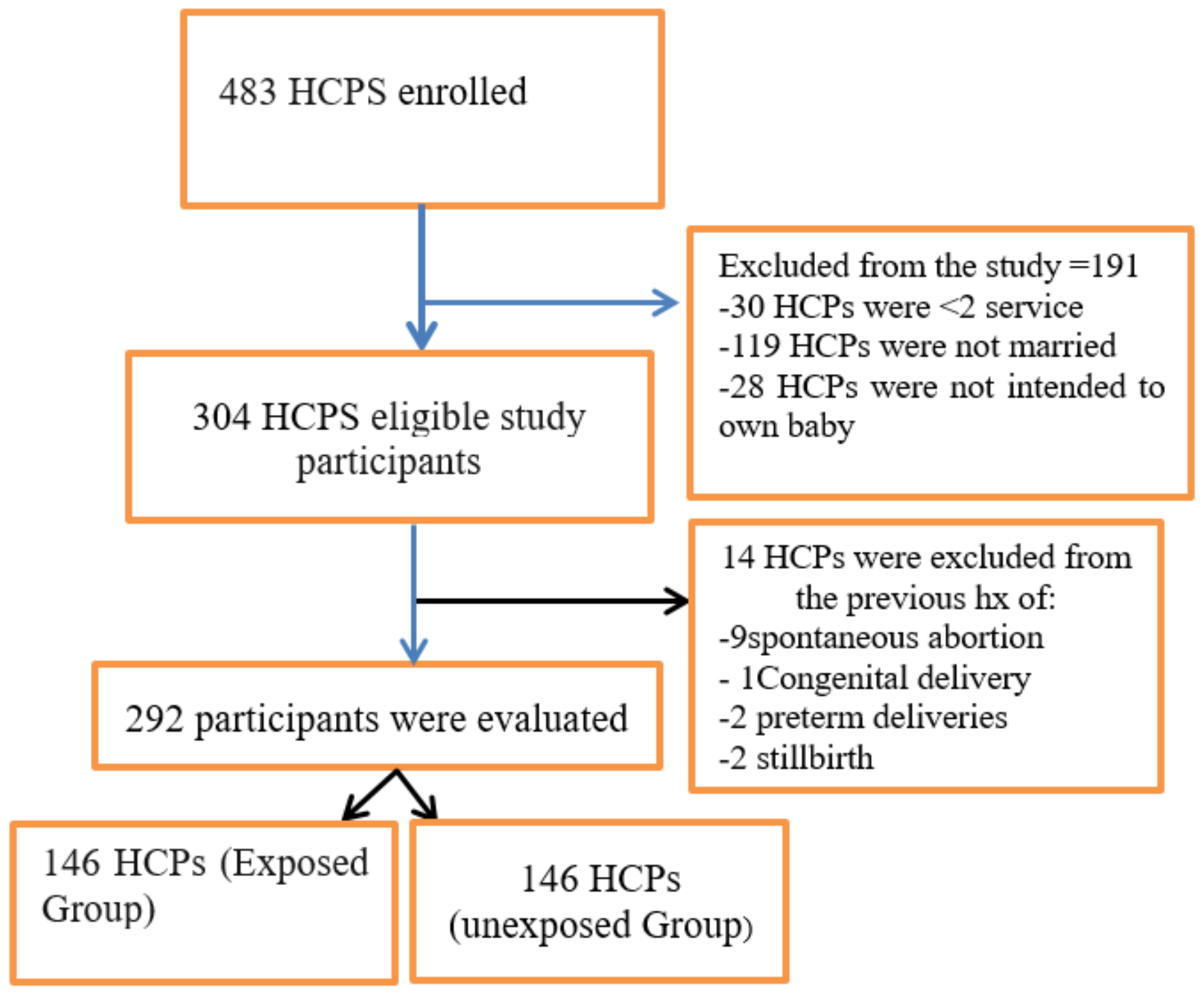

Figure 1

Flow chart depicting reasons for research participant's exclusion 


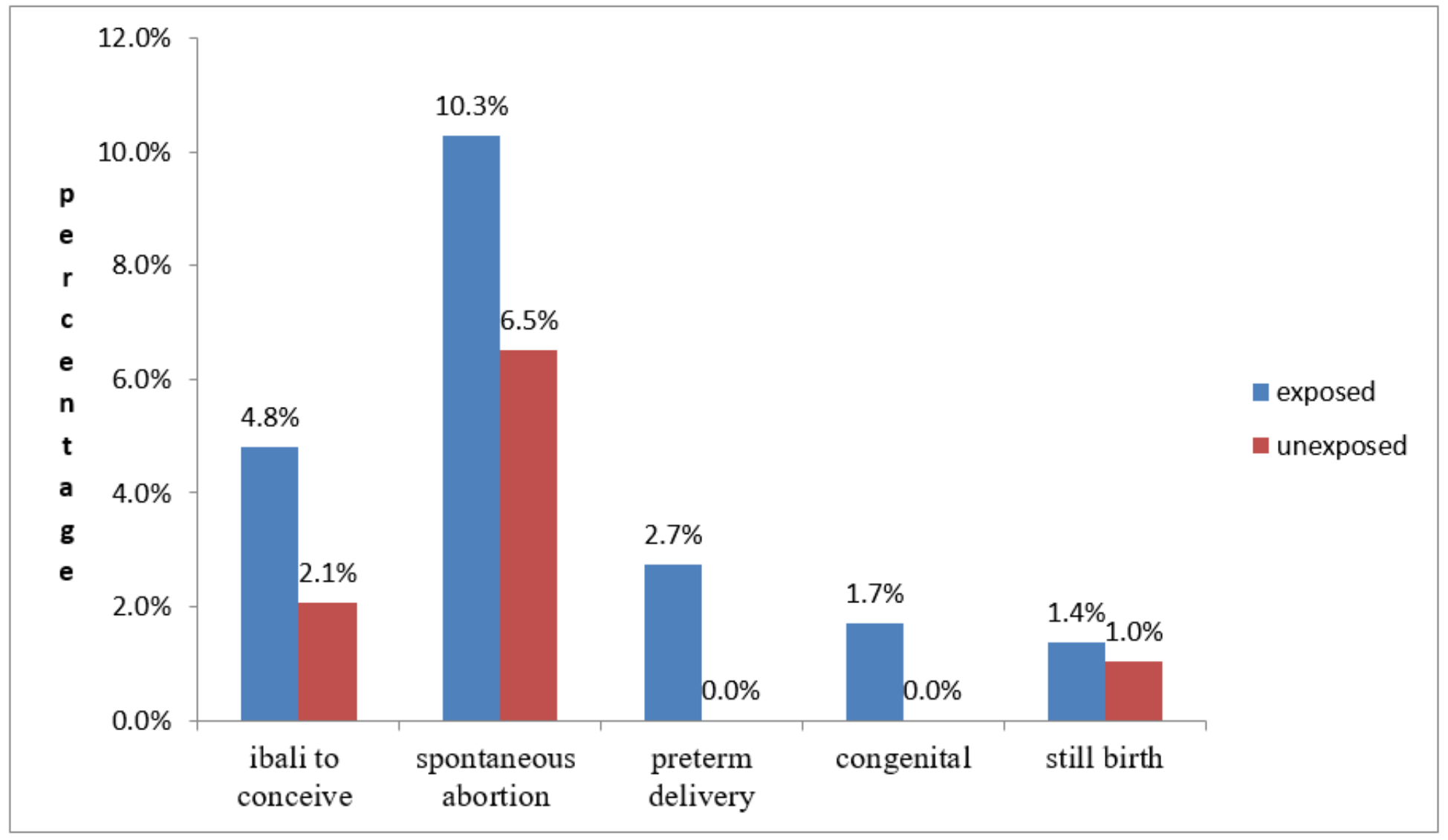

Figure 2

specific reproductive outcome status among health care personnel in Jimma zone public hospitals, southwest Ethiopia, 2021 


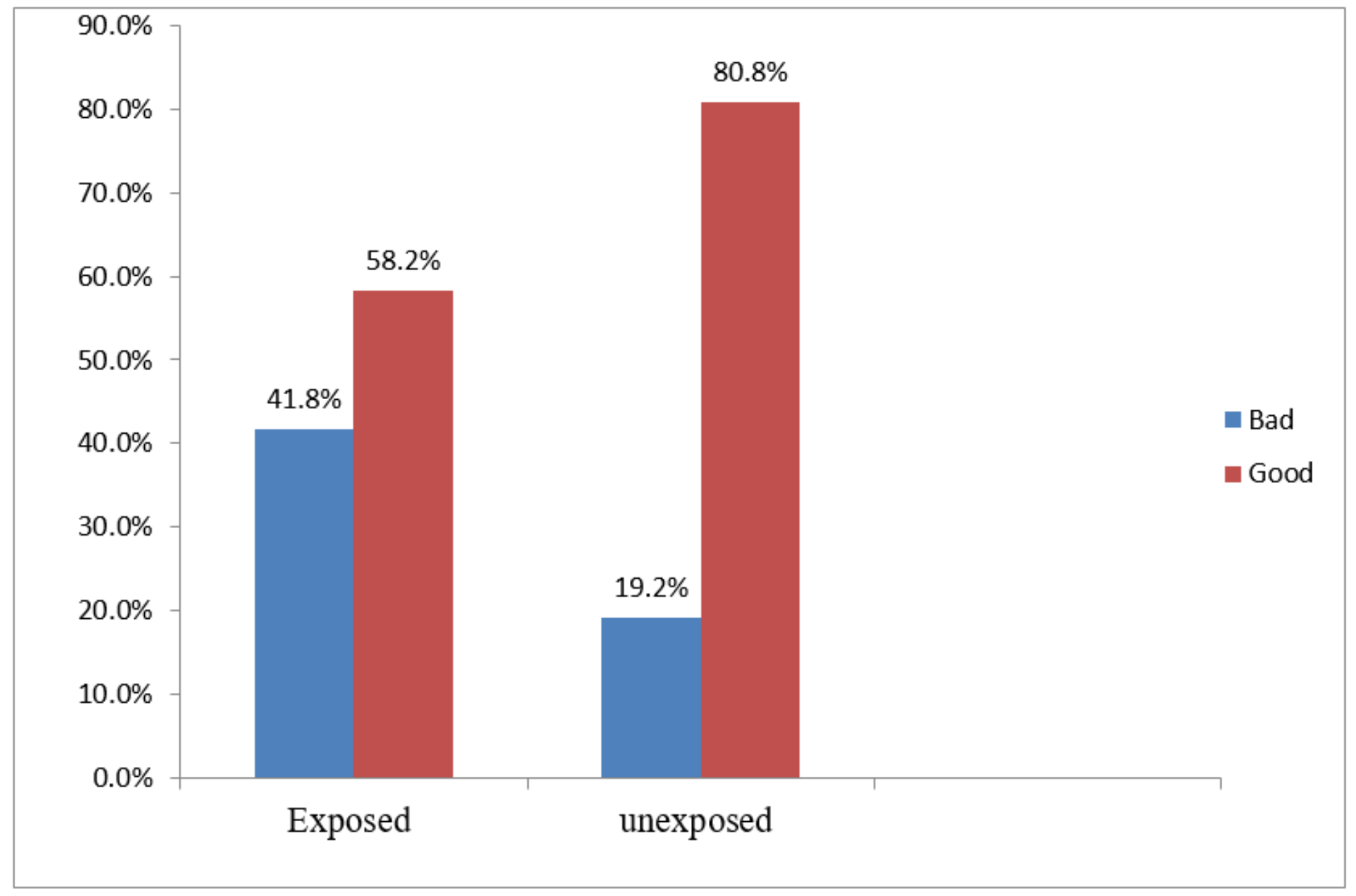

\section{Figure 3}

The comparison of reproductive outcome status among health care personnel in Jimma zone public hospitals, southwest Ethiopia, 2021. 